\title{
Visual processing during short-term memory binding in mild Alzheimer's disease
}

Gerardo Fernández $^{(a)\left(^{*}\right)}$, David Orozco ${ }^{(b)}$, Osvaldo Agamennoni ${ }^{(a)}$, Marcela Schumacher $^{(a)}$, Silvana Sañudo ${ }^{(a)}$, Juan Biondi ${ }^{(a)}$, and Mario A Parra ${ }^{(c, d)}$

$\left(^{a}\right)$ Universidad Nacional del Sur (UNS), Bahía Blanca, Argentina, Instituto de Investigaciones en Ingeniería Eléctrica (IIIE) (UNS-CONICET), Bahía Blanca, Buenos Aires, Argentina.

( ${ }^{b}$ ) Clínica Privada Bahiense, Bahía Blanca, Buenos Aires, Argentina.

(c) Department of Psychology, School of Social Sciences, Heriot-Watt University, Edinburgh, $U K$.

(d) Universidad Autónoma del Caribe, Facultad de Psicología, Barranquilla, Colombia.

$\left(^{*}\right)$ Corresponding author: Gerardo Fernández - IIIE - San Andrés 800 - 8000 Bahía Blanca, Buenos Aires, Argentina - Phone: 54-291-45951801 ext. 3321. e-mail: gerardo.fernandez@uns.edu.ar. 


\section{ABSTRACT}

Patients with Alzheimer disease (AD) typically present with attentional and oculomotor abnormalities that can have an impact on visual processing and associated cognitive functions. Over the last few years, we have witnessed a shift towards the analyses of eye movement behaviors as a means to further our understanding of the pathophysiology of common disorders such as AD. However, little work has been done to unveil the link between eye moment abnormalities and poor performance on cognitive tasks known to be markers for AD patients, such as the short-term memory-binding task. This was the aim of the present study. We analyzed eye movement fixation behaviors of thirteen healthy older adults (Controls) and thirteen patients with probable mild AD while they performed the visual shortterm memory binding task (Parra et al., 2011). The short-term memory binding task asks participants to detect changes across two consecutive arrays of two bicolored object whose features (i.e., colors) have to be remembered separately (i.e., Unbound Colors), or combined within integrated objects (i.e., Bound Colors). Patients with mild AD showed the well-known pattern of selective memory binding impairments. This was accompanied by significant impairments in their eye movements only when they processed Bound Colors. Patients with mild AD remarkably decreased their mean gaze duration during the encoding of color-color bindings. These findings open new windows of research into the pathophysiological mechanisms of memory deficits in $\mathrm{AD}$ patients and the link between its phenotypic expressions (i.e., oculomotor and cognitive disorders). We discuss these findings considering current trends regarding clinical assessment, neural correlates, and potential avenues for robust biomarkers. 
Keywords: visual short-term memory binding; Alzheimer's disease; eye movements; gazing; visual processing

\section{INTRODUCTION}

Visual information is thoroughly processed during fixations. Fixations normally last between $150 \mathrm{~ms}$ and $300 \mathrm{~ms}$ [1] and are driven by saccades which direct the fovea towards a particular element of interest [2]. Fixations are therefore the end result of complex interactions between features of the explored environment ("bottom up") and the instruction or question to be solved by the explorer ("top down") $[3,4,5,6,7,8,9,10]$. Behind such eye movement patterns, there are complex cognitive functions such as attention, executive control, and working memory $[11,12,13,14,15,9,16,17]$. Hence, the investigation of abnormal patterns of eye movement could provide critical information on the influence that some pathologies causing visual impairments, such as Alzheimer Disease (AD), have on these cognitive abilities, and in doing so unveil the source of capacity loss linked to specific processing levels.

In $\mathrm{AD}$ patients, progressive neuropathological changes affect certain laminae and cell types within the neocortex, which may lead to cortico-cortical disconnections $[17,18,19]$. This pathology, preferentially involves temporo-parietal association areas [20, 21, 22], whose damage could account for some of the differences found between AD patients and controls while performing cognitive tasks requiring perceptual organization, contrast sensitivity, spatial reasoning, and object recognition [23]. That AD patients are less able to process visual information is a finding well supported by the literature [24, for a review]. Subtle alterations in eye movement coordination and planning have been reported during performance on fine 
motor tasks from the initial course of the disease [25]. It has been suggested that the fixation network is affected in AD patients [24]. Impairments of such a network could result in delayed target visualization, probably due to altered projections from the frontal lobes to the superior colliculus [26]. As suggested by previous research [27, 28, 29, 30], a reduced activity in the fixation pathway could give rise to erroneous gaze shifts in $\mathrm{AD}$ patients. It seems that neurons in the rostral pole of the superior colliculus, which are active during visual fixation, become inhibited during gaze shifts in AD patients. This finding suggests that AD may cause a loss of ability to disengage fixation from the stimulus due to a failure of processes responsible for inhibiting fixation neurons. These studies demonstrated that patients with AD show altered visual search strategies and eye movement behaviors, with deficits in smooth pursuit eye movements, an increased number of saccades as well as increased attentional deficits and eye blinks [31, 32, 18, 33]. Porter et al. [34] showed that altered patterns of eye movements in AD patients may account for limitations to organize strategies during visual search tasks that rely on binding functions.

The present study compares visual processing in AD patients and healthy older adults (controls) during the short-term memory (STM) binding task. The STM binding task is a suitable tool to investigate the interplay of the above mentioned mechanisms and their potential link to eye movement disorders. The STM binding task is a change detection paradigm which assesses the ability to temporarily hold in memory either single features or features bound within integrated objects [35]. The task provides a measure of feature binding by contrasting performance during a condition in which features need to be remembered together (Bound Colors condition) relative to a baseline condition during which features are held in memory separately (Unbound Colors condition) [36, 35]. STM binding, as assessed by this paradigm, entails cortico-cortical interactions between areas responsible for 
processing features and those responsible for keeping them together in STM [37, 38]. STM binding declines in patients with dementia due to $\mathrm{AD}$ [39] and in those who will inevitably develop dementia due to familial $\mathrm{AD}$ but are still asymptomatic as demonstrated by traditional neuropsychological tasks [39]. STM binding is an integrative memory function known to support the conjunction of features necessary to create objects' identity [40]. Such a function relies on regions along the visual ventral stream but is independent of the hippocampus [41, 42]. In a recent work investigating structural connectivity, Parra et al. [43] showed that white matter integrity in frontal regions and in the anterior part of the corpus callosum accounted for a significant proportion of variance of STM binding performance in patients with familial AD. Moreover, Parra and collaborators recently showed that STM binding deficits in patients in the prodromal stages of familial AD are associated to altered patterns of functional (i.e., EEG) brain connectivity $[44,45]$ which seem to involve not only parietal-occipital regions but also the frontal lobes [46, 47]. The behavioral and neuroanatomical evidence gathered so far with STM binding tests lends support to the notion that this form of binding (i.e., conjunctive binding abilities) supports the temporary retention of multifeatured objects and reflects neither perceptual binding abilities nor hippocampal mediated memory functions. For instance, Parra and collaborators reported that all the AD patients assessed in their series of studies had intact perceptual binding functions confirmed by a screening tool used to assess binding in perception $[48,39]$. Further support to this view comes from the study by Parra et al. [41] in which they assessed healthy young volunteers while they perform a visual STM binding task similar to that reported here within the fMRI scanner. fMRI data revealed activation of regions within the visual ventral stream (i.e., Lateral Occipital Complex - LOC) during feature encoding. However, temporary memory for feature bindings activated similar regions of the LOC and also parietal and frontal lobe 
regions, thus confirming the reliance of such memory function on a dedicated brain network. In none of the above discussed studies was the hippocampus found to contribute to conjunctive binding functions carried out in visual STM. This evidence warrants investigation of the link between eye movements and visual STM binding as the network components which are specific to the latter function seem to be shared with the network supporting the former function (i.e., frontal-parietal network $[49,50])$. To date, there is no information available as to whether STM binding deficits and eye movement behaviors may be features of the clinical phenotype of $\mathrm{AD}$ patients, which could share pathophysiological mechanisms.

Investigating eye movement fixations in $\mathrm{AD}$ patients during STM binding performance would shed new light on the contribution of modulations of overt attention mechanisms across the different stages of memory processing (i.e., encoding and retrieval) to those impairments found with this task. Relying on the above discussed literature, two predictions are put forward regarding the contribution of the study of eye movements to cognition in AD. One (a) is linked to the role of memory load [51]. The STM binding task controls for this factor as the Bound and Unbound Color conditions present the same amount and type of features, and only the need of binding them together differs across these conditions. Hence, altered patterns of eye movements would be informative of limited binding abilities and not of overall processing deficits. The second (b) relates to the memory stages whereby the deficit could result from either poor encoding or impaired retrieval functions. Previous literature suggests that $\mathrm{AD}$ affects primarily memory encoding functions (see also [43, 44]). Fixation duration at these memory stages would inform on the extent to which eye movement impairments could be linked to poor encoding abilities or ineffective retrieval strategies. 


\section{METHODS}

\subsection{Participants}

Data were acquired from 13 controls (all native Spanish speakers) with mean age of 68 years $(\mathrm{SD}=4.2)$ and mean education of 18.2 (in years) and from 13 patients with $\mathrm{AD}$ (all native Spanish speakers) with mean age 67 ( $\mathrm{SD}=2.6$ years) and mean education 13.4 (in years). It is worth noting that the STM binding task has proved insensitive to the level of education of the assessed individual [40], a feature that has led to the suggestion that this task is a transcultural cognitive marker for AD [47]. Patients were diagnosed based on the Diagnostic and Statistical Manual of Mental Disorders (DSM-IV) criteria which were applied by a psychiatrist. They were recruited at the Hospital Municipal and at Clinica Privada Bahiense, both of Bahía Blanca, Buenos Aires, Argentina. All AD patients underwent a detailed clinical interview asking about medical history, physical/neurological examination and thyroid function test. All the patients underwent biochemical analysis (hemoglobin, full blood count, erythrocyte sedimentation rate, urea and electrolytes, blood glucose), to rule out other common pathologies. Patients were excluded if: (1) they suffered from any medical conditions other than dementia that could account for, or interfere with, their cognitive functioning; (2) had evidence of vascular lesions in computed tomography or RMN; (3) had evidence for an Axis I diagnosis (e.g. major depression or drug abuse) as defined by the DSM-IV. To be eligible for the study -if applicable-, patients had to have at least one caregiver providing regular care and support. Patients taking cholinesterase inhibitors (ChEI) were not included. None of the subjects was taking hypnotics, sedative drugs or major tranquillizers. Those participants with a diagnosis of Ophthalmologic diseases such as glaucoma, visually significant cataract or macular degeneration were excluded from the study. Subjects' visual acuity was $20 / 20$ or corrected to $20 / 20$. The investigation adhered to 
the principles of the Declaration of Helsinki. All patients and all control subjects signed an informed consent prior to their inclusion in the study.

The mean score of AD patients in the Mini-Mental State Examination (MMSE) [51] was 22.6 $(\mathrm{SD}=4.1)$ thus suggesting mild dementia. The mean score of AD patients in the Adenbrook's Cognitive Examination - Revised (ACE-R) [52] was 65.3 (SD = 16.9).

\subsection{The STM binding task and eye movement assessment}

Stimuli were presented on the center of a 20" LCD Monitor (1024 x 768 pixels resolution). Participants sat at a distance of $60 \mathrm{~cm}$ from the monitor. Head movements were minimized using a chin rest. Eye movements were recorded with an EyeLink 1000 Desktop Mount (SR Research) eyetracker, with a sampling rate of $1000 \mathrm{~Hz}$ and an eye position resolution of 20s arc. All recordings and calibration were binocular.

Participant's gaze was calibrated with a standard 13-point grid for both eyes. After validation of calibration, the STM binding task began. During the task, participants were presented with arrays of object shapes in random positions of a $3 \times 3$ virtual grid which sustained $10^{\circ}$ of visual angle. Objects were constructed using six different layouts, each defined by a shape and a frame area (see Fig. 1; also see [40]). The shape or frame area of each object (each representing $50 \%$ of the surface) was filled with a color. The procedures used to select the colors and the psychophysical features of the colors selected were reported in [40] and can be found in supplementary materials at http://www.era.lib.ed.ac.uk/bitstream/1842/2441/1/08-278-MAP.doc. During the task, participants were presented with arrays of two object shapes. Based on the study by Parra et 
al., [40] this seems an optimal array size to identify STM binding impairments in AD patients (see also [53]).

Trials began with a fixation screen (i.e., a cross) shown for $250 \mathrm{~ms}$. This was followed by a study display presented for $2000 \mathrm{~ms}$ (Fig. 1). After an unfilled interval of $900 \mathrm{~ms}$, the test display was presented until the participant responded. There was then an inter-trial interval of $1000 \mathrm{~ms}$. In half of the trials objects on both displays were the same. In the other half, the objects in the test display showed different colors from those in which they were presented during the study display. Object locations in the test display were always randomly changed to make location an uninformative feature. Participants were requested to detect whether the study and test displays consisted of the "same" or "different" items and to respond verbally accordingly. Responses were manually typed by a trained instructor.

Two experimental conditions were used (Fig. 1). In the Unbound Color condition both the shape and frame areas of each object were shown in different colors. In the "different" trials the color from either the shape $(50 \%)$ or the frame $(50 \%)$ area of the two objects was replaced by a new color that had not appeared in the study display. Participants were told to focus on colors and not on their associations as the change would consist of new colors. In the condition assessing memory for Bound Colors both the shape and frame area were also of different colors. However, in the "different" trials the two objects swapped either the color from the shape area $(50 \%)$ or from the frame area (50\%). Participants were told that colors and their associations were both relevant as sometimes colors would be rearranged in different combinations during the test display. For each condition participants performed 15 practice trials followed by 32 test trials. The "same" and "different" trials were fully randomized and conditions were blocked and counterbalanced across participants 
Insert Fig 1 about here

\subsection{Statistical analysis}

Statistical analyses were performed in R version 3.1.1 (RDevelopment Core Team). Group differences in the STM binding task were tested with ANOVA and linear regression. A model with saccade amplitude was created for each task to test for the relationship between these variables and task performance. We used the lmer program of the lme4 package (version 0.999999-2) [54] for estimating fixed and random coefficients. This package is supplied in the R system for statistical computing (version 3.1.1; R Development Core Team, 2013). We chose gaze duration (i.e., sum of consecutive fixation durations within a target) as the dependent variable because this measure includes refixations on a place, and refixations usually reflect a processing difficulty [1]. In addition, we transformed the dependent variable to $\log$ because it allowed us to obtain a normal distribution of residuals and a better statistical power. Fixed effects in LMM terminology correspond to regression coefficients in standard linear regression models. They can also estimate slopes or differences between conditions. We estimated how strongly the mean log gaze duration varied within participants by fitting a random intercept for participants. Instead of estimating a slope or the difference between conditions, random effects estimate the variance that is associated with the levels of a certain 
factor (i.e., participants' processing rate). Regression coefficients ( $b s$ ) standard errors (SEs) and t-values $(t=b / S E)$ are reported for the LMMs. Since there is no clear definition of "degree of freedom" for LMMs, precise $p$-values cannot be reported. The $t$-distribution is equivalent to the normal distribution for all practical purposes (i.e., the contribution of the degrees of freedom to the test statistics is negligible). Our criterion for referring to an effect as significant is $t=b / S E> \pm 1.95$.

\section{RESULTS}

\subsection{Correct recognition during the STM binding task}

Mean behavioral data during the STM binding task is shown in Figure 2. Relative to Controls, patients with $\mathrm{AD}$ showed a large drop in performance on the Bound Colors (BC) condition (66\% and $90 \%$ of correct responses for $\mathrm{AD}$ patients and controls, respectively) a discrepancy not found during the Unbound Color (UC) condition (87\% and 93\% of correct responses for AD patients and controls, respectively) (See Figure 2). To analyze performance, a two-way mixed ANOVA was used. The between-subject factor was Group (Controls vs. AD patients) and the within-subjects factor was Condition (BC vs. UC). The ANOVA resulted in a main effect of Condition: $(F=97.04, \mathrm{p}<0.001)$ but not of Group: $(F=3.04$, ns $)$. The Condition by Group interaction was significant $(F=13.32, \mathrm{p}<0.001)$. Four post-hoc comparisons, two across groups (i.e., UC: AD vs. controls and BC: AD vs. controls) and two across conditions (AD: UC vs. BC and Controls: UC vs. BC) were carried out to further investigate the interaction (corrected p-value $=0.005)$. They revealed that $\mathrm{AD}$ performed worse in the $\mathrm{BC}$ than in the UC condition ( $\mathrm{t}=4.23, \mathrm{p}=0.001)$. In addition, $\mathrm{AD}$ performed worse than controls in the $\mathrm{BC}$ than in the $\mathrm{UC}$ condition $(\mathrm{t}=4.51, \mathrm{p}=0.001)$. None of the other contrasts across 
groups or conditions resulted in significant differences. These results mirror those reported by Parra et al. [40].

\subsection{STM binding and gaze duration}

In Table 1 we report, (a) effects related to the mean values of gaze duration when averaging over all predictors; (b) interactions of Condition x Group, and (c) those interactions of Condition x Group x Memory Stage (Encoding vs. Retrieval).

Averaging over all predictors. We first evaluated mean log gaze duration as function of $\%$ of correct recognition. As shown in Table 1, \% of Correct Recognition as a function of gaze duration was non-significant $(t=1.34)$, but the effect trended toward the expected positive direction: gaze duration increases led to increased correct recognition. Saccade amplitudes exerted a significant effect on gaze duration $(t=-3.25)$ (See Table 1). When we analyzed the effect of Memory Stage (Encoding vs. Retrieval) collapsing across Group and Condition, we noted a significant effect on gaze duration, reflecting longer gazing times during Retrieval than during Encoding ( $t=2.26)$. Finally, when we analyzed the effect of Memory Stage and task Condition on gaze duration, we found significantly longer gazing times during Retrieval of BC than during retrieval of UC ( $t=3.27)$ (See Table 1).

Interaction of Group $x$ Condition. We then evaluated whether gaze duration was differentially affected during the $\mathrm{BC}$ condition when comparing controls and AD patients. As shown in Table 1 and in Figure 3, the mean gaze duration significantly decreased in AD patients with respect to controls $(\mathrm{t}=-3.51)$. However, when analyzing the UC condition, controls and AD patients showed similar gaze durations, which did not result in significant differences between groups $(t=0.22)$ (See Table 1 and Figure 3$)$. 
Interactions of Condition x Group x Memory Stage. Then we considered gaze duration as function of task Condition across Group and Memory Stage. As it is shown in Table 1, only during the $\mathrm{BC}$ task there was a significant effect between groups ( $t=2.38)$, whereby Controls and AD patients showed well differentiated gaze durations. While controls showed a similar gaze duration between Encoding and Retrieval, AD patients showed significantly shorter gaze duration during Encoding than during Retrieval (See slope for controls and AD patients during Encoding and Retrieval on the $\mathrm{BC}$ condition in Figure 3. More details about this striking effect are presented in the Discussion section). In contrast, both groups showed similar gaze durations when performing the UC task ( $t=1.60)$ (See Figure 3).

\section{DISCUSSION}

Given that the $\mathrm{BC}$ and $\mathrm{UC}$ condition presented the same amount and type of features, and only the need of binding them together differed across these conditions; we investigated whether subtle changes in binding processing such as those presented in mild AD patients might coexist with abnormal eye movements, detectable by an eyetracker coupled to the STM task. Our results provide evidence that even at these early stages of the disease, AD patients showed gross changes during eye movements fixations associated to the $\mathrm{BC}$ condition of the STM binding task, compared to healthy controls. This suggests that the analysis of eye movements might provide an additional useful tool to identify early clinical expressions in $\mathrm{AD}$ patients which are associated to impairments of cognitive functions known to hold marker properties for this type of dementia (i.e., the STM binding test). As shown in Figure 3, controls' encoding responses differ from those seen in $\mathrm{AD}$ patients. Controls seem to meet 
the encoding demands of the $\mathrm{BC}$ task more effectively than $\mathrm{AD}$ patients. $\mathrm{AD}$ patients however, showed shorter gaze duration particularly during the encoding stage of the $\mathrm{BC}$ condition. This suggests that poor encoding mechanisms could be the source of impairments found in these patients during STM binding tasks. Similar conclusions were presented by Parra et al [see 43] and Pietto et al. [46] using brain connectivity and ERP analysis. A reduction in gaze duration and in the number of fixations during the encoding of bound information may be the output of inefficient cortical integration mechanisms responsible for forming or holding the master map of locations which, as suggested by Treisman and Gelade [55], would keep features together in perception and seemingly in STM. Such a map will bind together features sharing the same visual space. The fact that such a pattern of impairments was not observed during the UC condition which presented identical number and type of feature rules out differential visuospatial demands of the $\mathrm{BC}$ condition as a potential driver of this effect. This leaves binding as the potential candidate which could account for the altered pattern of eye movements found in AD patients during the STM binding task reported here. There is evidence that AD patients can bind surface features in perception [39], particularly when these features are processed within the same visual stream (i.e., ventral as it is the case for the stimuli presented here [56]). This suggests that AD patients may have specific problems encoding visual bindings in STM seemingly due to poor cognitively guided visual commands. Daffner et al. [57] showed that AD patients exhibit a diminished visual curiosity when identifying novel stimulus, probably due to impaired neural mechanisms responsible for controlling the cortical components of the motivational drive. However, the results presented here would be unlikely accounted for by lack of visual curiosity. The fact that the UC and BC presented identical visual materials and that the function found to be impaired (i.e., binding) is rather a construct which need to be applied to 
succeed in this task, make the visual curiosity hypothesis an unlikely account. Nevertheless, Daffner et al. [57] proposed that visual curiosity impairments in AD patients could be mediated by dysfunction of prefrontal association cortices. Such cortical regions also play a role in STM binding functions. Hence, future studies will need to investigate the extent to which impaired eye movements, altered STM binding functions, and reduced visual curiosity in $\mathrm{AD}$ may share neurocognitive mechanisms.

Notwithstanding these knowledge gaps, the results presented here suggest that eye movements may be a sensible approach to collect subjects' performance when analyzing visual information, from the encoding through the retrieval stages of memory. AD patients and controls had longer and similar fixations when encoding UC targets (see Figure 3), being our results compatible with previous evidence about relatively preserved feature processing in mild AD patients [42]. Parra et al. [40] showed that processing BC demanded more cognitive resources than the $\mathrm{UC}$, and that this seems to be true regardless of age [58]. However, previous evidence suggests that AD selectively impairs feature binding [40]. Interestingly, our study shows that gaze duration follows a pattern of impairment similar to that observed through other behavioral measures of STM performance (e.g., percentage of correct recognition), thus suggesting a strong link between these two neurocognitive responses.

A fMRI study reported binding-specific activation in posterior parietal regions $[50,59,60$, 61] which are also known to be part of the network supporting top-down attentional and eye movement control [62]. Moscovitch [63] suggested that the inferior parietal lobe mediates the automatic allocation of attention to retrieved memory contents. Petersen and Posner [48] proposed that overt shifts of attention through eye movements are associated with higher 
accuracy of performance in relational visuospatial memory task. Others $[64,65,66]$ have argued that the activation of these parietal lobe regions may reinforce and help to produce stronger spatial representations and, consequently, stronger visualization would produce more accurate memory recognitions. The last decade has witnessed a surge of studies documenting a parietal dysfunction in AD patients which seems to be associated to reduced exploration strategies $[67,18]$. In our study, controls' and AD patients' saccade amplitudes did not show significant differences through the BC and UC conditions of the STM tasks. More research will be needed to understand saccade behaviors during the STM binding task reported here.

In summary, we propose that the analysis of eye movements during STM binding tasks provides a valuable measure to further assess disease mechanisms. Eye movement disorders reflect deficits in attention and working memory processes in AD patients. We suggest that a more comprehensive evaluation of eye movements during binding, incorporating both an in-depth analysis of oculomotor responses and assessment of cognitive processes, may well provide a user-friendly marker of early disease symptoms and future progression. Future longitudinal studies can investigate whether abnormal eye movement responses linked to STM binding impairments can reliably anticipate the diagnosis of AD. Moreover, future studies should refine our understanding of the interplay between eye movements and the cognitive constructs underlying memory binding during STM tasks. Particular emphasis should be placed on the causal relationship between these phenotypic features of the most common form of dementia.

\section{ACKNOWLEDGMENTS}


This work was supported by Consejo Nacional de Investigaciones Científicas y Técnicas (CONICET) to Dr. Gerardo Fernández and by the Universidad Nacional del Sur and CIC to Dr. O. Agamennoni. Mario A Parra is supported by Alzheimer's Society (Grant AS-SF-14008). We acknowledge the support from the University of Edinburgh's Centre for Cognitive Ageing and Cognitive Epidemiology, part of the UK cross council Lifelong Health and Wellbeing Initiative (MR/L501530/1). Funding from the Biotechnology and Biological Sciences Research Council (BBSRC) and Medical Research Council (MRC) is gratefully acknowledged. We acknowledge the support from Alzheimer's Scotland Dementia Research Centre also from the University of Edinburgh. 


\section{REFERENCES}

[1] Rayner K. (1998). Eye movements in reading and information processing: 20 years of research. Psychological Bulletin. 124:372-422.

[2] Martinez-Conde S, Macknik SL, Hubel DH (2004). The role of fixational eye movements in visual perception. Nature Neuroscience. 5:229-240.

[3] Awh E, Vogel EK, Oh SH. (2006). Interactions between attention and working memory. Neuroscience. 139:201-208.

[4] Cowan N, Morey CC. (2006). Visual working memory depends on attentional filtering. Trends in Cognitive Sciences. 10,139-141.

[5] Gilbert CD, Sigman M. (2007). Brain states: Top down influences in sensory processing. Neuron. 54:677-696.

[6] Khayat PS, Spekreijse H, Roelfsema PR. (2006). Attention lights up new object representations before the old ones fade away. Journal of Neurosciences. 26:138-142.

[7] Palmer J. (1998). Attentional limits on the perception and memory of visual information. Journal Experimental Psychology: Human Performance. 16:332-350.

[8] Sigman M, Gilbert CD Learning to find a shape (2000). Nature Neuroscience. 3:264-269.

[9] Yarbus A. (1967). Eye Movements and Vision. Plenum. New York.

[10] Fernández G, Laubrock J, Mandolesi P, Colombo O, Agamennoni O. (2014a). Registering eye movements during reading in Alzheimer disease: difficulties in predicting upcoming words. Journal of Clinical and Experimental Neuropsychology. 36:302-316 
[11] Hayhoe M, Ballard D. (2005). Eye movements in natural behavior. TRENDS in Cognitive Sciences. Vol.9 No.4.

[12] Hoffman J. (1998). Visual attention and eye movements. In H. Pashler (ed.), Hove, UK: Psychology Press, pp. 119-154.

[13] Itoh N, Fukuda T. (2002). Comparative study of eye movement in extent of central and peripheral vision and use by young and elderly walkers. Perceptual Motor Skills, 94(3 Pt 2), 1283-91.

[14] Miela D, Lobel E, Lehericy S, Pierrot-Deseilligny C, Berthoz A. (2005). Cortical mechanisms of saccadic generation from execution to decision. Annals New York Academy of Sciences. 1039:232-8.

[15] Posner M. (1980). Orienting of attention. Quarterly Journal of Experimental Psychology, 32:3-25.

[16] Fernández G, Schumacher M, Castro L, Orozco D, Agamennoni O. (2015). Patients with Alzheimer disease produced shorter outgoing saccades when reading sentences. Psychiatry Research. 229:470-478.

[17] Grady CL, Furey ML, Pietrini P, Horwitz B, Rapoport SI. (2001). Altered brain functional connectivity and impaired short-term memory in Alzheimer's disease. Brain. 124: 739-56. 
[18] Mosimann UP, Felblinger J, Ballinari P, Hess CW, Müri RM. (2004). Visual exploration behavior during clock reading in Alzheimer's disease. Brain. 127:431-438.

[19] Fernández G, Manes F, Rotstein N, Colombo O, Mandolesi P, Politi L, Agamennoni O. (2014b). Lack of contextual-word predictability during reading in patients with mild Alzheimer disease. Neuropsychologia. 62:143-151

[20] Levine DN, Lee JM, Fisher CM. (1993). The visual variant of Alzheimer's disease: a clinicopathologic case study. Neurology. 43:305-313.

[21] Hendrie HC. (1998). Epidemiology of dementia and Alzheimer's disease. Journal Geriatric Psychiatry. 6:3-18.

[22] Wong AMF. (2008). Eye Movement Disorders. New York: Oxford University Press. p. $165-177$.

[23] Cronin-Golomb A, Gilmore GC, Neargarder S, Morrison SR, Laudate TM. (2007)

Enhanced signal strength improves visual cognition in aging and Alzheimer's disease. Cortex, 43(7), $952-966$.

[24] Pereira M, Camargo M, Aprahamian I, Forlenza O. (2014) Eye movement analysis and cognitive processing: detecting indicators of conversion to Alzheimer's disease. Neuropsychiatric Disease and Treatment. 10:1273-1285.

[25] Molitor RJ, Ko PC, Ally BA. (2015). Eye movements in Alzheimer's disease. J. Alzheimer's Dis. 44, 1-12. 10.3233/JAD-141173

[26] Crawford, T. J. et al. (2005). Inhibitory control of saccadic eye movements and cognitive impairment in Alzheimer's disease. Biological Psychiatry 57, 1052-1060. 
[27] Abel, L. A., Unverzagt, F. \& Yee, R. D. (2002). Effects of stimulus predictability and interstimulus gap on saccades in Alzheimer's disease. Dementia Geriatry Cognitive Disorders. 13, 235-243.

[28] Munoz DP, Wurtz RH (1992). Role of the Rostral Superior Colliculus in Active Visual Fixation and Execution of Express Saccades. Journal of Neurophysiology. 67:1000-1002. [29] Munoz DP, Wurtz RH (1993a): Fixation Cells in Monkey Superior Colliculus .1. Characteristics of cell discharge. Journal of Neurophysiology. 70:559 -579. [30] Munoz DP, Wurtz RH (1993b): Fixation Cells in Monkey Superior Colliculus .2. Reversible Activation and Deactivation. Journal of Neurophysiology. 70:576 -589.

[31] Braak H, Thal DR, Ghebremedhin E, Del TK. (2011). Stages of the pathologic process in Alzheimer disease: age categories from 1 to 100 years. Journal of Neuropathology and Experimental Neurology. 70, 960-969.

[32] Lueck K, Mendez M, Perryman. (2000). Eye movements abnormalities during reading in patients with Alzheimer disease. Neuropsy Neuropsy Be. 13(2):77-82.

[33] Fernández G, Mandolesi P, Rotstein N, Colombo O, Agamennoni O, Politi L. (2013). Eye movement alterations during reading in patients with early Alzheimer Disease. Invest. Ophthalmology and Visual Sciences. doi:10.1167/iovs.13-12877.

[34] Porter G, Leonards U, Wilcock G, Haworth J, Troscianko T, Tales A. (2010) New insights into features and conjunction search: II. Evidence from Alzheimer's disease. Cortex. $46,637-649$. 
[35] Wheeler ME, Treisman AM. (2002). Binding in short-term visual memory. Journal of Experimental Psychology: General. 131(1):48-64.

[36] Olson I, Jiang Y. (2002). Is visual short-term memory object based? Rejection of the “strong-object" hypothesis. Perception \& Psychophysics. 64(7):1055-1067.

[37] Smith K, Azami H, Escudero J, Parra MA, Starr JM. (2015). Comparison of Network Analysis Approaches on EEG Connectivity in Beta during Visual Short-Term Memory Binding Tasks. In (pp. 2207-2210).

[38] Ibañez A, Parra MA. (2014). Mapping memory binding onto the connectome's temporal dynamics: toward a combined biomarker for Alzheimer's disease. Frontiers Human Neurosciences. 8:237.

[39] Parra MA, Abrahams S, Logie RH, Mendez LG, Lopera F, Della Sala S. (2010). Visual short-term memory binding deficits in familial Alzheimer's disease. Brain. 133: 2702-2713.

[40] Parra MA, Sala SD, Abrahams S, Logie RH, Mendez LG, Lopera F. (2011). Specific deficit of colour-colour short-term memory binding in sporadic and familial Alzheimer's disease. Neuropsychologia, 49:1943-1952.

[41] Parra MA, Della Sala S, Logie RH, Morcom AM. (2014). Neural correlates of shapecolor binding in visual working memory. Neuropsychologia. 52: 27-36.

[42] Parra et al., (2015). Memory binding and white matter integrity in familial Alzheimer's disease. Brain. 138(5):1355-69.

[43] Parra MA, Mikulan E, Trujillo N, Sala SD, Lopera F, Manes F. et al. (2017). Brain information sharing during visual short-term memory binding yields a memory biomarker 
for familial Alzheimer's disease. Current Alzheimer Research, 14, 1335-1347 doi: $10.2174 / 1567205014666170614163316$

[44] Olsen RK, Lee Y, Kube J, Rosenbaum RS, Grady CL, Moscovitch M. et al. (2015). The role of relational binding in item memory: evidence from face recognition in a case of developmental amnesia. Journal of Neuroscience, 35:5342-5350.

[45] Smith, K., Ricaud, B., Shahid, N., Rhodes, S., Starr, J. M., Ibañez, A. et al. (2017). Locating Temporal Functional Dynamics of Visual Short-Term Memory Binding using Graph Modular Dirichlet Energy. Scientific Reports, 7, 42013.

[6] Pietto, M., Parra, M. A., Trujillo, N., Flores, F., Garcia, A. M., Bustin, J. et al. (2016). Behavioral and Electrophysiological Correlates of Memory Binding Deficits in Patients at Different Risk Levels for Alzheimer's Disease. Journal Alzheimers Disease. 53, 1325-1340.

[47] Della Sala, S., Kozlova, I., Stamate, A., \& Parra Rodriguez, M. (2016). Temporary memory binding: A transcultural cognitive marker of Alzheimers Disease. International Journal of Geriatric Psychiatry.

[48] Petersen, S. E. \& Posner, M. I. (2012). The attention system of the human brain: 20 years after. Annual Review of Neuroscience, 35, 73-89.

[49] Corbetta, M., Kincade, J. M., \& Shulman, G. L. (2002). Neural systems for visual orienting and their relationships to spatial working memory. Journal of Cognitive Neuroscience.14(3):508-23. 
[50] Azuma M, Minamoto T, Yaoi K, Osaka M, Osaka N. (2014). Effect of memory load on eye movement control: A study using the reading span test. Journal of Eye Movement Research. 7(5).

[51] Folstein MF, Folstein SE, McHugh PR. (1975). `Mini-mental state'. A practical method for grading the cognitive state of patients for the clinician. J Psychiatr Res ,12:189-98.

[52] Mioshi E, Dawson A, Mitchell J, Arnold R, Hodges JR. (2006). The Addenbrooke's Cognitive Examination Revised (ACE-R): a brief cognitive test battery for dementia screening. Int J Geriatr Psychiatry, 21(11):1078-85.

[53] Della Sala S, Kozlova I, Stamate A, Parra MA. (2017). A transcultural cognitive marker of Alzheimer's Disease. International Journal of Geriatric Psychiatry, n/a.

[54] Bates D, Maechler M. (2013). lme4: Linear mixed-effect models using S4 classes R package versión 0.995-2.

[55] Treisman, A. M. \& Gelade, G. (1980). A feature-integration theory of attention. Cognitive Psychology. 12, 97-136.

[56] Festa EK, Insler RZ, Salmon DP, Paxton J, Hamilton JM, Heindel WC. (2005). Neocortical disconnectivity disrupts sensory integration in Alzheimer's disease. Neuropsychology. 19: 728-738.

[57] Daffner, KR., Scinto, LF., Weintraub, S., Guinessey, B.S., Mesulam, M.M. (1992). Diminished curiosity in patients with probable Alzheimer's disease as measured by exploratory eye movements. Neurology. 42, 320-328.

[58] Parra, M. A., Abrahams, S., Logie, R., \& Della Sala, S. (2009). Age and binding withindimension features in visual short term memory. Neuroscience Letters. 449, 1-5. 
[59] Xu Y, Chun MM. (2006). Dissociable neural mechanisms supporting visual short-term memory for objects. Nature. 440:91-95.

[60] Shafritz KM, Gore JC, Marois R. (2002). The role of the parietal cortex in visual feature binding. Proc.Natl.Acad.Sci.U.S.A, 99:10917-10922.

[61] Todd JJ, Marois R. (2005). Posterior parietal cortex activity predicts individual differences in visual short-term memory capacity. Cogn Affect.Behav.Neurosci. 5:144-155.

[62] Gazzaley, A. (2013). Top-down Modulation Deficit in the Aging Brain: An Emerging Theory of Cognitive Aging. Stuss, D.T.\& Knight, R.T. (Eds.), Principles of Frontal Lobe Function, 2nd ed., p.593-608.

[63] Moskovitch M. (2008). The Hippocampus as a "stupid," domain-specific module: implications for theories of recent and remote memory. Canadian Journal of Experimental Psychology. 62:62-69.

[64] Cummings JL, Houlihan JP, Hill MA. (1986). The pattern or reading deterioration in dementia of the Alzheimer's type: observations and implications. Brain Lang. 29:315-23. [65] Kennedy A, Pynte J, Murray W, Paul S. (2012). Frequency and predictability effects in the Dundee corpus. Quarterly Journal of Experimental Psychology. doi.10.1080/17470218.2012.676054.

[66] Fernández G, Laubrock J, Mandolesi P, Colombo O, Agamennoni O. (2014a). Registering eye movements during reading in Alzheimer disease: difficulties in predicting upcoming words. Journal of Clinical and Experimental Neuropsychology. 36:302-316 
[67] Rizzo M, Anderson SW, Dawson J, Myers R, Ball K. (2000). Visual attention impairments in Alzheimer's disease. Neurology. 54:1954-9. 
Table 1: Parameter estimates for fixed effects of Linear Mixed Models. Threshold of significance is set at $\mathrm{t}= \pm 1.95$.

\begin{tabular}{|lccc|}
\hline & \multicolumn{3}{c|}{$\begin{array}{c}\text { Gaze } \\
\text { duration }\end{array}$} \\
\cline { 2 - 4 } Fixed effects & S & t-value \\
Mean Gaze duration (log) & 6.668 & 0.060 & $\mathbf{1 1 0 . 0 2}$ \\
\% of Correct Recall & 0.007 & 0.005 & 1.34 \\
Saccade amplitude & -0.009 & 0.030 & $\mathbf{- 3 . 2 5}$ \\
Encoding vs. Recognition & 0.035 & 0.015 & 2.26 \\
UC vs. BC X Encoding vs. Recognition & 0.523 & 0.159 & $\mathbf{3 . 2 7}$ \\
\hline Group x Task Condition & & & \\
Control vs. AD X BC & -0.467 & 0.133 & $\mathbf{- 3 . 5 1}$ \\
Control vs. AD X UC & 0.027 & 0.125 & 0.22 \\
Group x Task Condition x Memory Stage & & & \\
(Encoding vs. Retrieval) & & & \\
Control vs. AD X BC X Enc. vs. Rec. & 0.271 & 0.114 & $\mathbf{2 . 3 8}$ \\
Control vs. AD X UC X Enc. vs. Rec. & 0.181 & 0.113 & 1.60 \\
\hline Variance components & & Variance & SD \\
Subject & & 0.035 & 0.187 \\
Residual & & 0.072 & 0.269 \\
\hline
\end{tabular}




\section{FIGURE CAPTIONS}

Figure 1. Trial design for the two conditions of the STM tasks.

Figure 2. Corrected recognition during the two experimental conditions in both controls and $\mathrm{AD}$ patients (error bars $=$ standard errors of the mean).

Figure 3. Effect of binding task on gaze duration in control and in Alzheimer Disease (AD) patients during Encoding and Recognition moments. .Panel show partial effects of LMM (i.e., after removal of other fixed effects and variance components). Bar errors denote 95\%confidence intervals. Gaze duration is plotted on a log scale for correspondence with the LMM. 
Unbound Colours

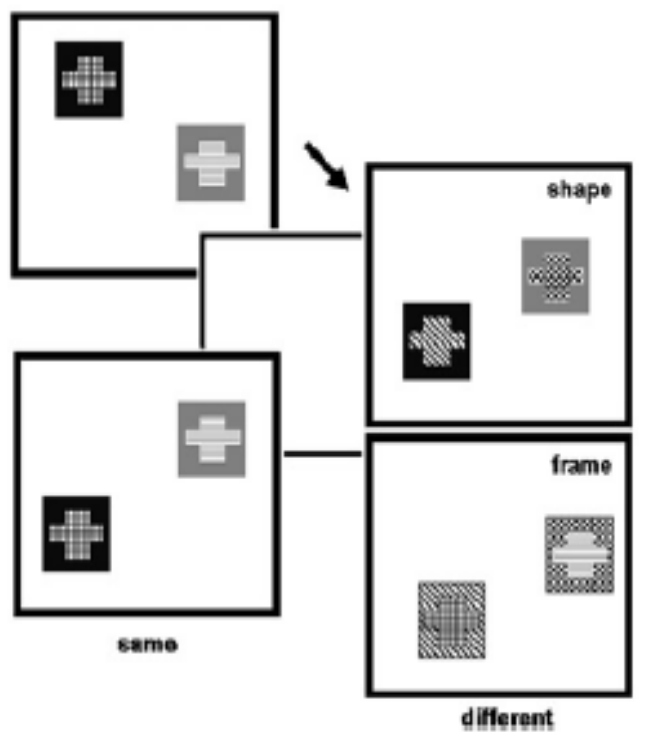

100

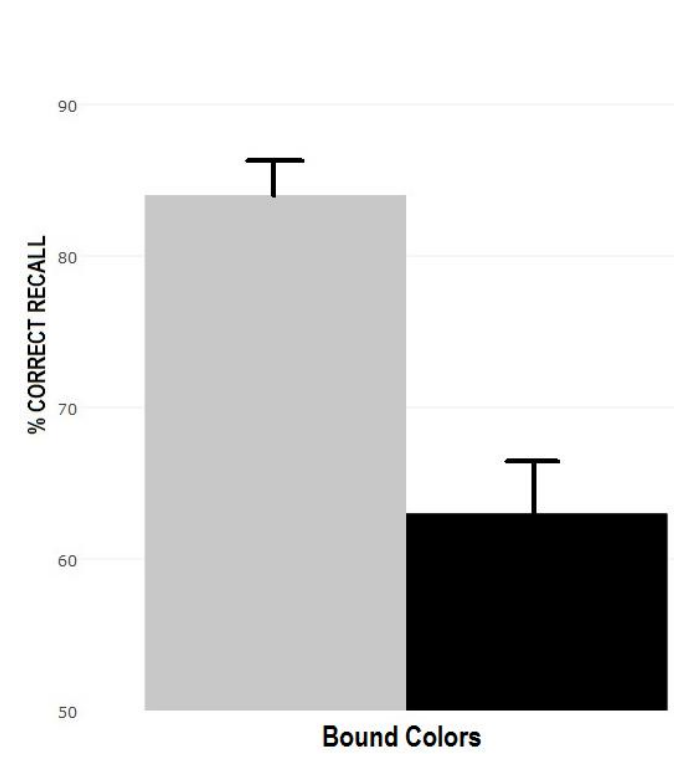

\section{Bound Colours}

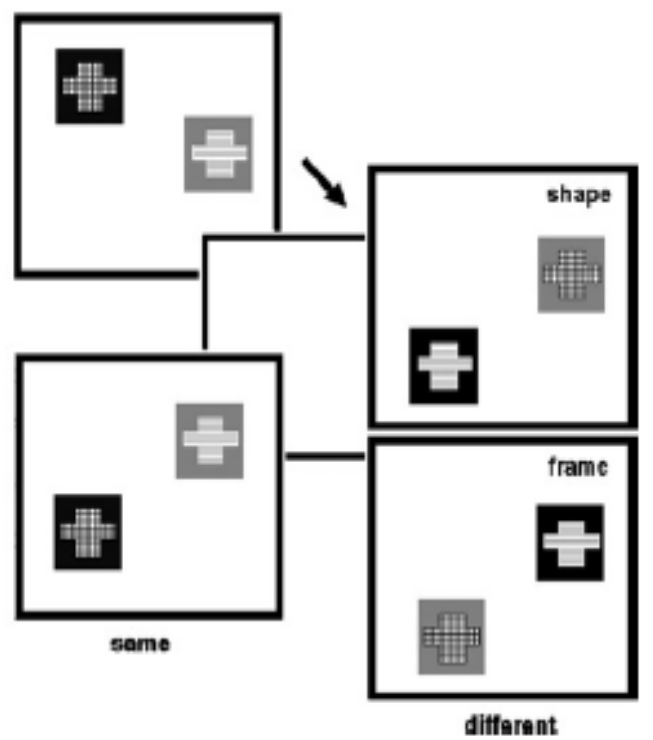

Figure 2

BINDING TASK

CONTROLS
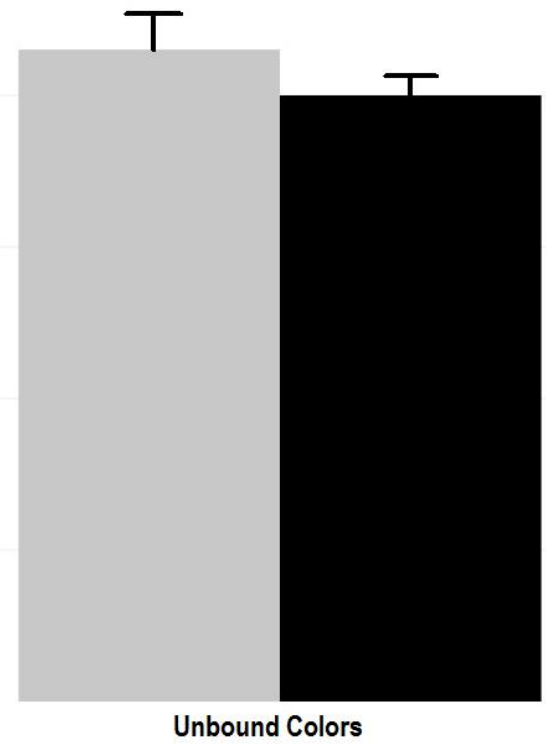
Figure 3

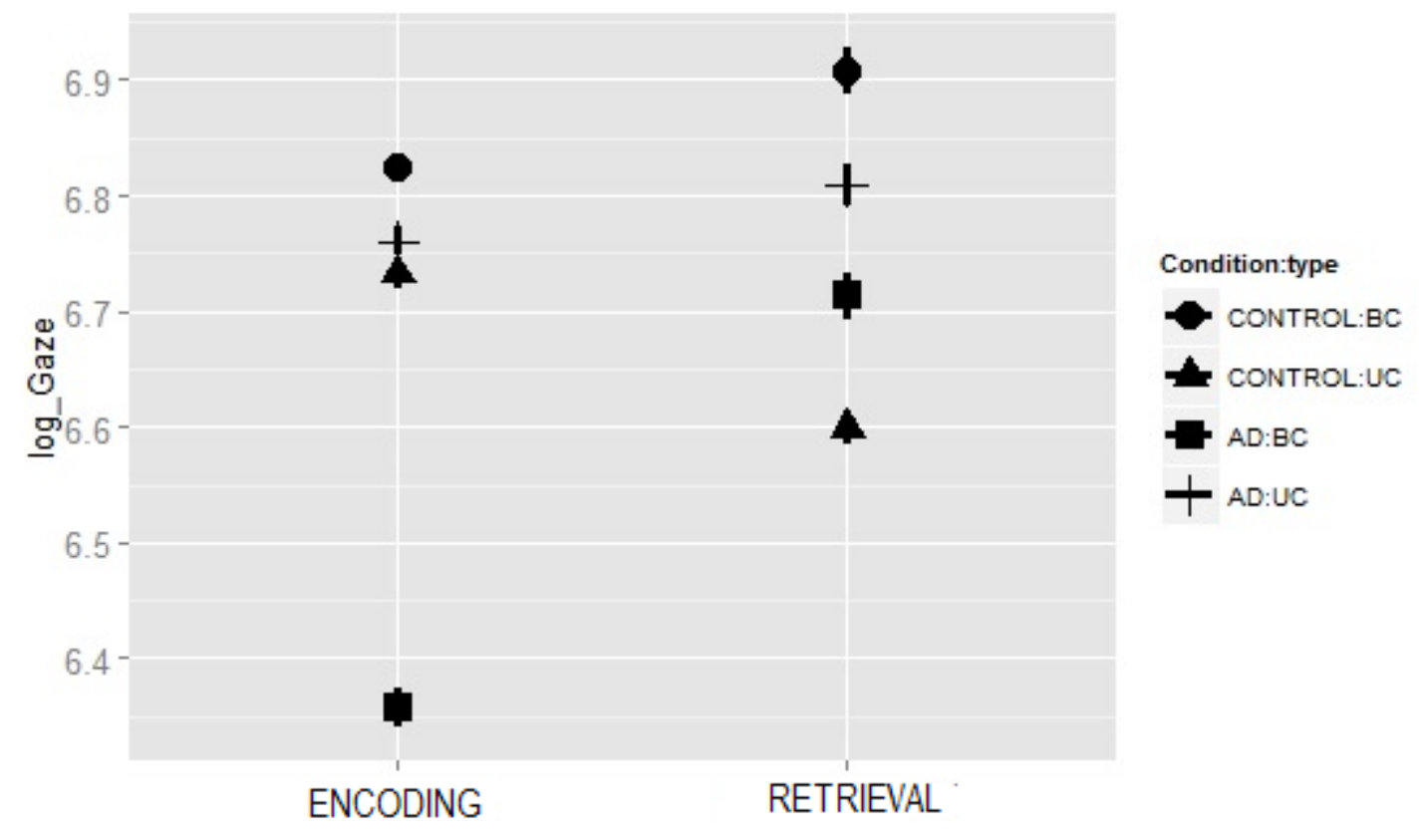

